\title{
Structural and Optical Properties of Pure Wurtzite ZnO under Uniaxial Strain Based on First-Principles Study
}

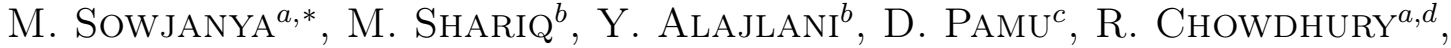 \\ R. JAYAGANTHAN ${ }^{a, e}$ AND S.M. TAQIULLAH ${ }^{b}$ \\ ${ }^{a}$ Centre of Nanotechnology, Indian Institute of Technology Roorkee, Roorkee 247 667, India \\ ${ }^{b}$ Department of Physics, Faculty of Science, Jazan University, Jazan 45142, Saudi Arabia \\ ${ }^{c}$ Department of Physics, Indian Institute of Technology Guwahati, Guwahati 781 039, India \\ ${ }^{d}$ Department of Civil Engineering, Indian Institute of Technology Roorkee, Roorkee 247667, India \\ ${ }^{e}$ Department of Metallurgical and Materials Engineering, Indian Institute of Technology Roorkee, \\ Roorkee 247667, India
}

\begin{abstract}
(Received September 2, 2019; revised version November 11, 2019; in final form November 20, 2019)
First-principles computation is used to examine lattice parameters, energy band gap and optical properties of $\mathrm{ZnO}$ under uniaxial strain. The calculation is carried out in uniaxial ( $c$-axis) strain changing in the range from $-5 \%$ to $5 \%$. The simulations are based upon the Perdew-Burke-Ernzerhof form of generalize gradient approximation within the density functional theory. There is linear variation of lattice constant and linear boarding of energy band gap with uniaxial strain increment. Strain in the $c$-axis direction has prime contribution in the variation of energy band of $\mathrm{ZnO}$. Absorption coefficient, optical properties comprising dielectric function (real and imaginary) and reflectivity of wurtzite $\mathrm{ZnO}$ are deduced. Diminishing of imaginary part of the dielectric function with the increase of uniaxial strain at low energy is observed in calculated results but imaginary part of the dielectric function is observed to increase with increasing uniaxial strain for energy greater than $5.0 \mathrm{eV}$ and a blue shift is appeared in graph. Strained band gap of $\mathrm{ZnO}$ is larger than that of unstrained $\mathrm{ZnO}$, which provides the theoretical reference value for the modulation of the band gap of $\mathrm{ZnO}$.
\end{abstract}

DOI: 10.12693/APhysPolA.137.361

PACS/topics: uniaxial strain, first-principles, optical properties, $\mathrm{ZnO}$

\section{Introduction}

Recently, zinc oxide ( $\mathrm{ZnO})$, a II-VI semiconductor compound has attracted incredible attention for researchers. It has high excitation binding energy of $60 \mathrm{meV}$ and large energy band gap of $3.37 \mathrm{eV}$ at normal temperature $[1,2]$. $\mathrm{ZnO}$ based derivatives have been used for many years for different applications, such as LCD screens, solar cells, gas sensors, detectors, flat panel displays and ultraviolet semiconductor laser beam. It is nontoxic and has piezoelectric, ferroelectric, and ferromagnetic properties $[3,4]$. There are three established polymorphs of $\mathrm{ZnO}$ : (i) wurtzite ( $P 63 / m c$ space group), (ii) zinc blende $(F 43 m)$, and (iii) rocksalt $(F m / 3 m)$. Wurtzite structure is thermodynamically steadier at encompassing conditions. Each sublattice incorporates four atoms per unit cell in wurtzite structure of $\mathrm{ZnO}$. Atom of $\mathrm{Zn}$ (group II) is encompassed by 4 atoms of the $\mathrm{O}$ (group $\mathrm{VI}$ ), or the other way around [5].

Several methods were utilized to produce $\mathrm{ZnO}$ thin films such as RF magnetron sputtering, chemical vapor deposition, sol-gel, spray-pyrolysis and pulsed laser deposition [6]. Stress-strain produced by lattice mismatch between the film and substrate, and thermal mismatch

*corresponding author; e-mail: sowjiphysics@gmail.com created by various thermal expansion coefficients are unavoidably present in the thin films [7]. Quality of crystal and optical execution of $\mathrm{ZnO}$ film are influenced by this mismatch. Consequently, it is imperative to examine variation of lattice constant, band gap, and absorption coefficient of $\mathrm{ZnO}$ films under application of strain for the arrangement of research study on epitaxial layers and hetero intersections. Recent analysis of some physical properties of $\mathrm{ZnO}$ under uniaxial strain has pulled in the broad enthusiasm of analysts. The Raman spectroscopy, X-rays diffraction, and other spectroscopic methods are utilized to quantify residual strain in films. First-principles estimations are also reported to examine physical mechanism of $\mathrm{ZnO}$ under the application of strain [8]. Alvaro et al. explored the structural and electronic and characteristics of nanoporous $\mathrm{ZnO}$ under biaxial stress, through first-principles methods based on total energy $a b$ initio calculations using spin-polarized density functional theory. In comparison with bulk, remarkable inverse pattern of tuning of energy band by the strain was reported and surfaces states in the nanoporous are answerable for a strange variation in the energy gap [9]. Shao et al. studied transport properties of $n$-type $\mathrm{ZnO}$ nanowires utilizing the in situ scanning tunneling microscope, transmission electron microscope technique and found continuous decrease of $\mathrm{ZnO}$ nanowires resistance with increasing compressive strain, but it increased under increased tensile strain. They additionally announced 
varieties of bandgaps during the application of tensile strain of individual $\mathrm{ZnO}$ nanowires via cathodoluminescence spectroscopy [10]. The first-principles hybrid density functional theory was used to investigate structural and electronic properties of wurtzite $\mathrm{ZnO}$ under hydrostatic strain and uniaxial strain along $c$-axis by Wang et al. Structural transformation of the wurtzite $\mathrm{ZnO}$ under tensile hydrostatic strain and compressive uniaxial strain is reported in this paper. They utilized generalized gradient approximation (GGA) for this work, as their focus was on relative variation of the band gap and the electronic effective mass in wurtzite $\mathrm{ZnO}$ under tensile hydrostatic strain and compressive uniaxial strain [11]. In spite of the fact that some ground progress of $\mathrm{ZnO}$ under stress-strain is studied, but execution of $\mathrm{ZnO}$ is still seldom announced [12]. First principle computation based on the density functional theory is also reported for band gap calculation and the optical properties of p-type $\mathrm{ZnO}$ under uniaxial strain $[13,14]$, which indicated large band gap of $\mathrm{ZnO}$ under strain. Present work investigates the optical properties of $\mathrm{ZnO}$ by optimization of the geometry and computation of first-principles approach. The band structure and optical properties of $\mathrm{ZnO}$ are studied and strong anisotropy is revealed. Additionally, energy band gap of $\mathrm{ZnO}$ is tuned continuously under uniaxial strain ( $-5 \%$ to $5 \%$ interval). The electronic dispersions of these systems have been calculated using the tight binding approximation. Evolutions of optical properties, for example, dielectric constant, refractive index and absorption coefficient were carried out by Green's functions. The current work will be helpful for encourage of experimental findings in near future. This paper is sorted out as pursues. Initially the computational methodology, the band gap details and theory are presented and secondly the key results of optical properties such as dielectric constant, absorption coefficient and refractive index are explained in sequential manner in results and discussion section. At last the main outcomes are compiled in conclusion section.

\section{Computational details}

Wurtzite structure of stable $\mathrm{ZnO}$ is a hexagonal with the space group of $P 63 / m c$ and $C 6 v-4$ symmetry. Unit cell specifications for this structure are $a=b=3.249 \AA$, $c=5.206 \AA, \alpha=\beta=90^{\circ}$, and $\gamma=120^{\circ}$ [15]. The calculations in the framework of density functional theory (DFT) based on first-principles calculations ( $a b$ initio calculations) were implemented in Atomistix Toolkit software (ATK). In this strategy, no shape estimates on either potential or the electronic charge density are based on the local density approximation of the DFT theory. However, reproducing electronic structure correctly is well known problem of DFT which occurs because of incorrect electron counting in DFT [16]. Consideration of ground state during the calculation in most of theories is one of big problem. Therefore, the effects of final state, configuration interactions, and variety of finite temper- ature effects etc. are normally not taken into consideration by theory [17]. Further, due to complication in many calculations, the $4 f$ and $5 f$ shells are considered as shallow cores but experimentally these levels have band structure. Hedin suggested GW approximation, which is one of successful attempts to go beyond DFT/LDA [18]. There is estimate of the self-energy operator by utilizing Green's function $G$ for the electron system, in the screened Coulomb field $W$. Accurate estimation of the band gap can be executed by GW approximation but it is time consuming because of its complexity. A new version (PW91) of the generalized gradient approximation (GGA) was introduced by Perdew and Wang in 1991 [19]. Improvements of LDA results for atoms, molecules, and solid surfaces were successfully exhibited by PW91. The latter value for the effective enhancement of the exchange potential was explored by Perdew, Burke and Ernzerhof (PBE) and has become the standard of GGA, and implemented in most of the modern program packages. However, ground state properties of perovskite-type $\mathrm{ABO}_{3}$ materials were predicted accurately by first-principles method based on DFT within local density approximation [20]. Conventional DFT-LDA approach is not able to effectively describe the localization of strongly correlated $d$ and $f$ electrons and it gets collapse for strongly correlated transition material cases [21]. The hybrid DFT-HF functional (such as BH\&HLYP, B3LYP, and B3PW91) [22], dynamic mean field theory (DMFT) [23], and DFT-LDA+U functional [24] have successfully improved the calculation of localization of strongly correlated electrons in transition metal. Yaakob et al. [25] have explored semiempirical $\mathrm{LDA}+\mathrm{U}$ approach for treatment of transition metal of $\mathrm{Zn} 3 d$ electron with different values of effective Hubbard $U$ potential.

The Perdew-Burke-Ernzerhof (PBE) functional method in the generalized gradient approximation (GGA) was utilized for exchange-correlation functional [26, 27], since the GGA has produced important data about properties of material. However, a noted key limitation is that the GGA make band gaps considerably smaller than experiment [28]. Summations of band structure and optical spectrum program parameter calculations used in this work are based on DFT combined with double zeta polarized states. The ATK-DFT program can demonstrate the optical properties of shut and open quantum systems with DFT models utilizing numerical premise sets. Non-equilibrium Green functions are used to calculate density matrix for open systems. Similarly, for closed or periodic systems, selfreliable densities can be determined by resulting iterative diagonalization of the Kohm-Sham Hamiltonian, the Fermi-population of the Kohn-Sham states. Electron density can be characterized by density matrix, and the electron density decides about an effective potential, i.e. the Hartree and exchange-correlation potential. The Kohn-Sham Hamiltonian equation acquired from the effective potential in which the non-interacting particles movement occurs. DFT quantum mechanical 
modeling considers electronic structure of the system as one-electron Kohn-Sham Hamiltonian. Density mesh cut-off about $400 \mathrm{eV}$, electron temperature $300 \mathrm{~K}$ at $k$-point sampling $(4 \times 4 \times 4)$ was used. In distinctive characteristics of $\mathrm{ZnO}$ and its apllied materials, lattice strains incite effective gauge fields on the electronic carriers. Uniaxial strain ranging from $-5 \%$ to $5 \%$ was considered against band gap in this study.

Based on the first principle calculations using DFT, the uniaxial strain effect on energy band structure was studied. The energy band structure of simulation results showed that tensile strain caused a slight reduction in band gaps. At the same time small and large compressive strain induces slight increase and decrease of band gaps of electronic band structure which are reliant upon energy levels of the constituent atomic orbital and the crystal structure. The band structure graph includes data about bonding interactions inside a molecule. However, understanding capability of band structure diagrams enables us to separate profitable data about the material electronic conductivity, optical properties, and the strength oxidation or reduction of material. It is important to know optical behavior of metallic and nonmetallic materials in terms of their absorption, reflection and transmission characteristics in response to electromagnetic radiation and particular to visible light. The amount of energy absorbed by metals depends on the electronic structure of each metal. First-principle based on the DFT joined with the plane-wave pseudopotential method is executed for the calculation [29]. To simplify the calculation, ionic potential is portrayed by a Vanderbilt type ultrasoft pseudopotential to reduce the computation. $3 d^{10}, 4 s^{2}$, and $2 s^{2}, 2 p^{4}$, are taken as electronic configurations of $\mathrm{Zn}$ and $\mathrm{O}$ atoms, respectively. Computation is supposed to be non-spin polarized and generalized gradient approximation is utilized to handle exchange-correlation function. We mindfully tried the total energy relying upon cutoff energy and $k$-point examining, to be sure about convergence of calculation. Ghosh et al. have also reported about final cutoff energy [30]. Strain was directed along $c$-axis which set the uniaxial strain along the $c$-axis but strains along the $a$-axis and the $b$-axis were not limited.

\section{Results and discussion}

\subsection{Structural parameters}

The strain along the $c$-axis, $\varepsilon_{z z}$ is represented by the following expression:

$$
\varepsilon_{z z}=\frac{c-c_{0}}{c_{0}} \times 100 \%,
$$

where $c_{0}$ is lattice constant of $\mathrm{ZnO}$ without strain and $c$ is the lattice constant with strain. As per above relation tensile strain will be positive and compressive strain will be negative. Calculated values of $\varepsilon_{z z}$ utilizing above expression are mentioned in Table I. Uniaxial strain varies from minimum $-5 \%$ to the maximum strain value $5 \%$ corresponding to applied stress on $\mathrm{ZnO}$ film. Figure 1 shows calculated lattice constants $a$ and $c$ with variation

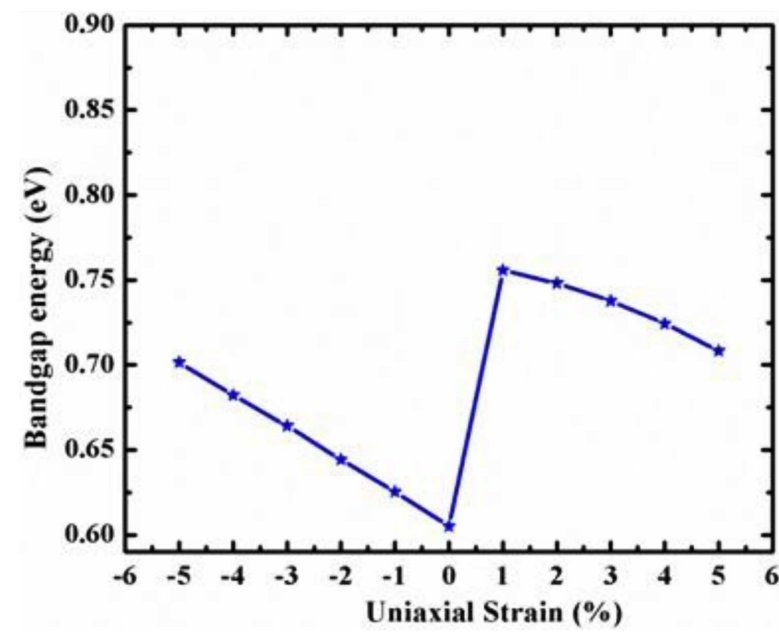

Fig. 1. Band gap of $\mathrm{ZnO}$ versus uniaxial strain (-5\% to $5 \%)$.

TABLE I

Lattice parameters, cell volume $V$ and band gap varying with uniaxial strain.

\begin{tabular}{c|c|c|c|c|c|c|c}
\hline \hline \multirow{2}{*}{ Strain } & \multicolumn{2}{|l|}{ Lattice parameters } & Volume & $\mathrm{u}$ & $\mathrm{L}$ & $\begin{array}{c}\text { Band } \\
\text { gap }\left(E_{g}\right)\end{array}$ \\
\cline { 2 - 6 }$a[\AA]$ & $c[\AA]$ & $c / a$ & $(V)$ & & & 0.701 \\
\hline-5 & 3.249 & 4.946 & 1.522 & 45.219 & 86.347 & 424.658 & 0.682 \\
-4 & 3.249 & 4.998 & 1.538 & 45.695 & 88.17 & 438.244 & 0.682 \\
-3 & 3.249 & 5.05 & 1.554 & 46.17 & 90.009 & 452.09 & 0.664 \\
-2 & 3.249 & 5.102 & 1.57 & 46.647 & 91.871 & 466.252 & 0.644 \\
-1 & 3.249 & 5.154 & 1.586 & 47.119 & 93.737 & 480.593 & 0.625 \\
0 & 3.249 & 5.206 & 1.602 & 47.598 & 95.647 & 495.426 & 0.605 \\
1 & 3.249 & 5.258 & 1.618 & 48.074 & 97.562 & 510.444 & 0.755 \\
2 & 3.249 & 5.311 & 1.634 & 48.55 & 99.5 & 525.792 & 0.748 \\
3 & 3.249 & 5.363 & 1.65 & 49.026 & 101.456 & 541.445 & 0.737 \\
4 & 3.249 & 5.415 & 1.666 & 49.502 & 103.429 & 557.373 & 0.724 \\
5 & 3.249 & 5.467 & 1.682 & 49.978 & 105.423 & 573.643 & 0.708
\end{tabular}

of uniaxial strain. Values of lattice constants without strain are $a=3.249 \AA$ and $c=5.206 \AA$, which are different from lattice constant values under uniaxial strain. It is clear from Fig. 2 that $c$ value varies linearly with strain and $a$ is almost constant. Lattice parameters, $c / a$ ratio and volume of unit cell under uniaxial strain are tabulated in Table I. It is obvious from Table I that $c / a$ ratio and volume of unit cell increases with increment of uniaxial strain and this is because of increasing value of $c$ with strain.

\subsection{Band gap}

Variation of energy band gap of $\mathrm{ZnO}$ with strain is revealed by Fig. 1. Computations were carried out using hexagonal cell of $\mathrm{ZnO}$ in different calculation. Applied uniaxial strain values were ranged from $-5 \%$ to $5 \%$ with step size of $1 \%$ and band gap were calculated at each step. The band gaps were computed through GGA-PBE and different trends were found. Energy band gap increases with compressive stain and $E_{g}$ is maximum 


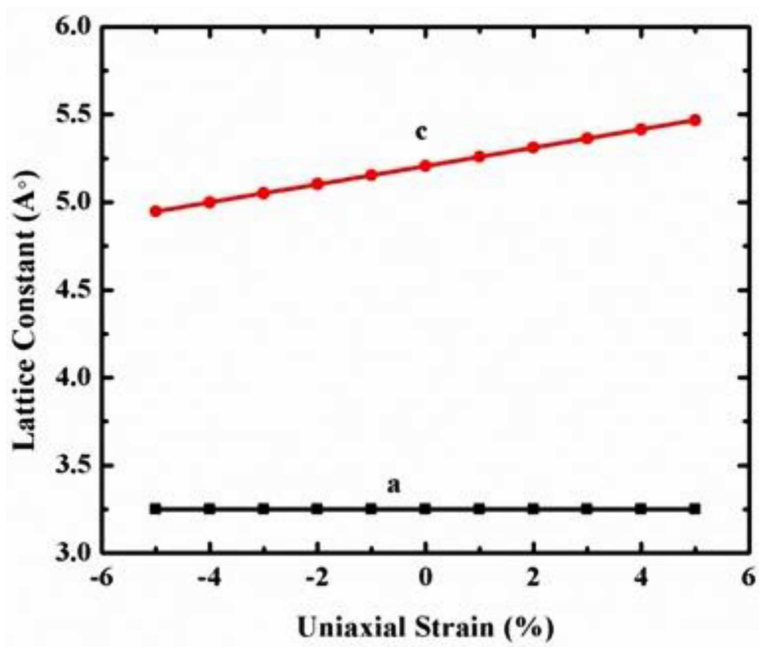

Fig. 2. Lattice constants $a$ and $c$ versus uniaxial strain of $\mathrm{ZnO}$ under different strain ( $-5 \%$ to $5 \%)$.

at $1 \%$ tensile strain which starts decreasing with tensile strain. Particularly, the energies of the conduction and valance bands at $\Gamma$ climb under compressive strain, while move down with tensile strain. There is prevailing effect of shifting energy levels on the bands around uniaxial strain, so it can tune curvature of the dispersion relation at the band edges. In this way we can expect modification of effective masses of the electron and the hole. Under compressive strain, the effective mass of the electron is increased, while the effective mass of the hole is diminished. In the contrast, under tensile strain, the effective mass of the electron is decreased. Therefore, step change of energy band might be due to reverse depending of electron effective mass on compressive strain and tensile strain [31]. Energy band gap of $\mathrm{ZnO}$ without strain is found to be $0.605 \mathrm{eV}$, which is in agreement with outcomes revealed by Liu et al., however, this value is significantly smaller than the experimental result. Smaller value of band gap in theoretical calculation is due to fact that GGA functional calculations generally give underestimated values of band gap [32]. It can be observed that there is linear broadening of band gaps with the increment of uniaxial strain. From Fig. 2, it is exhibited that under uniaxial strain, value of lattice constant $c$ diminishes by $4.85 \%$ compared to $c$ value without strain whereas $a$ is constant with uniaxial strain, and this is because of structural relaxation. Therefore, role of strain in the $c$-axis direction has main contribution for the variation of band gap of $\mathrm{ZnO}$, and this consists of affinity between lattice constant and energy band gaps of $\mathrm{ZnO}$ [33]. Band gap energy with different strain is given in Table I.

The band structure of $\mathrm{ZnO}$ near the $\Gamma$ point without strain is shown in Fig. 3. The same $k$ values in the Brillouin zone of bottom of the conduction band and top of the valence band confirm $\mathrm{ZnO}$ as direct band gap type semiconductor. Valence band maxima (VBM) and the conduction band minima $(\mathrm{CBM})$ are situated at the $\Gamma$
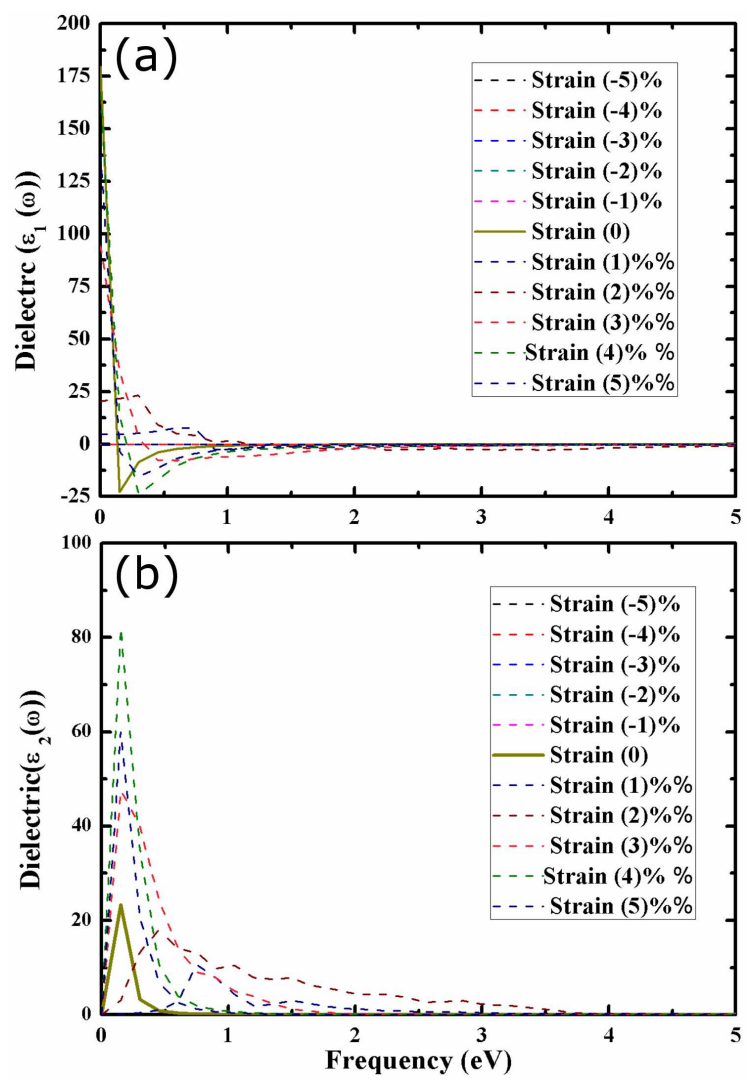

Fig. 3. Variations of the real (a) and imaginary (b) part of $\varepsilon_{1}(\omega)$ with frequency energy under uniaxial strain $(-5 \%$ to $5 \%)$.

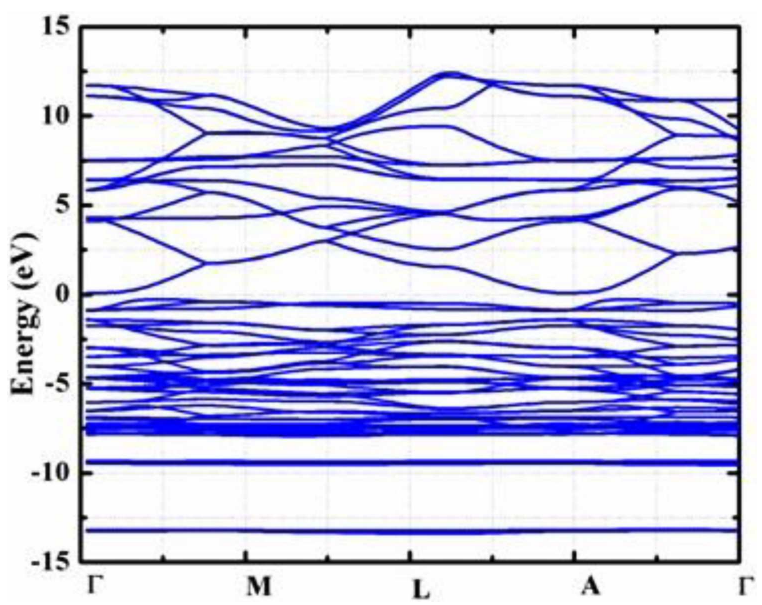

Fig. 4. VBM and CBM near the $\Gamma$ point $\mathrm{ZnO}$ unstrained condition.

point for direct band gap $\mathrm{ZnO}$ semiconductor. Representation of CBM and VBM by Fig. 4 indicates that the band gap of $\mathrm{ZnO}$ can be varied by strain. Therefore, analysis of VBMs and CBMs under increasing uniaxial strain has great importance for understanding of change of band gap under strain. 
There is significant shift of VBM toward the lower energy and marginal move of CBM toward lower energy with increasing uniaxial strain. The counterbalance of the VBM is more prominent over CBM shift, which legitimately creates the widening of energy band gap. Additionally, electrons on the bonding orbit of $\mathrm{Zn} 4 s$ have main contribution to form energy level $\Gamma$. Doublet $\Gamma$ controls VBM, which is mainly formed by electrons on the antibonding state $\mathrm{O}$ of $2 P$. Energy level $\Gamma$ in conduction band moves toward the higher energy whereas energy level $\Gamma$ in valance band moves oppositely by increasing uniaxial strain. This is because of electron scattering produced by uniaxial strain on the wurtzite semiconductor, creating valley deformation potential [34].

\subsection{Optical properties}

The optical properties are among the most attractive and functional properties of nanomaterials. These properties have been extensively studied using a variety of optical spectroscopic techniques. In the computational framework, calculations are performed within the projector augmented wave (PAW) $[35,36]$ method as implemented using ATK. In this area of work, the exchangecorrelation potential was described by meta-GGA functional TB09, given by Tran and Blaha [37] which is a semi-empirical functional and suitably fit for decent explanation of the band gaps for non-metals. Outcomes of advanced many-body calculations are generally comparable with this method. In this manner, the meta-GGA functional might be practically useful way for getting a decent depiction of the electronic structure. External electric field $E_{\text {ext }}$ gets coupled with the internal electric field (expecting from crystal feedback) by optical response functions. Displacement field $D$, has relation with internal electric field $E$ and polarization $P$, as below

$$
D=\varepsilon_{0} E+P .
$$

The polarization is defined as dipole moment per unit volume:

$$
P \equiv \frac{p}{V},
$$

where $V$ is the volume of the material. These quantities are related to susceptibility $\chi$, dielectric constant $\varepsilon_{r}$, and polarizability $\alpha$, through given expression

$$
P=\varepsilon_{0} \chi E, D=\varepsilon_{r} \varepsilon_{0} E, p=\alpha E .
$$

The Kubo-Greenwood formula is used to calculate the susceptibility tensor for estimating optical spectrum as given below

$\chi_{i j}(\omega)=-\frac{e^{2} \hbar^{4}}{m^{2} \varepsilon_{0} V \omega^{2}} \sum_{n m} \frac{f\left(E_{m}\right)-f\left(E_{n}\right)}{E_{m n}-\hbar \omega-i \hbar \Gamma} \pi_{n m}^{i} \pi_{n m}^{j}$,

where $\pi_{n m}^{i}, \pi_{n m}^{j}$ belong to $i$-th and $j$-th component of the dipole matrix element between state $n$ and $m$, respectively, $V$ is the volume of the material, $\Gamma$ is broadening, and $f$ indicates Fermi function in the expression. Dielectric constant $\varepsilon_{r}$, polarizability $\alpha$, and optical conductivity $\sigma$, have relation with susceptibility as described below

$$
\begin{aligned}
& \varepsilon_{r}(\omega)=(1+\chi(\omega)), \quad \alpha(\omega)=V \varepsilon_{0} \chi(\omega), \\
& \sigma(\omega)=-\mathrm{i} \omega \varepsilon_{0} \chi(\omega) .
\end{aligned}
$$

The refractive index $n$ has relation with complex dielectric constant through extinction coefficient $\kappa$ :

$$
n+\mathrm{i} \kappa=\sqrt{\varepsilon_{r}} .
$$

In terms of the real $\left(\varepsilon_{1}\right)$ and complex $\left(\varepsilon_{2}\right)$ parts of the dielectric constant, the refractive index $n$ and the extinction coefficient $\kappa$ are formulated as follows:

$$
\begin{aligned}
& n=\frac{\sqrt{2}}{2}\left[\sqrt{\varepsilon_{1}^{2}+\varepsilon_{2}^{2}}+\varepsilon_{1}\right]^{1 / 2}, \\
& \kappa=\frac{\sqrt{2}}{2}\left[\sqrt{\varepsilon_{1}^{2}+\varepsilon_{2}^{2}}-\varepsilon_{1}\right]^{1 / 2} .
\end{aligned}
$$

The optical absorption coefficient $\alpha$ and the reflectivity $r$ is related to the extinction coefficient and mathematically it can be written as

$$
\begin{aligned}
& \alpha_{a}=\frac{2 \omega \kappa}{c}, \\
& r=\frac{(1-n)^{2}+\kappa^{2}}{(1+n)^{2}+\kappa^{2}} .
\end{aligned}
$$

Therefore, many optical properties might be derived easily by real and imaginary parts of the diagonal components of the dielectric constant. Since $\mathrm{ZnO}$ has hexagonal symmetry, so isotropic dielectric constant i.e. $\varepsilon_{x x}=$ $\varepsilon_{y y}=\varepsilon_{z z}=0$ is considered for evaluating the optical properties. To investigate the optical characteristics of $\mathrm{ZnO}$, real and imaginary part of the dielectric function were calculated. Dielectric constant is one of the primary features of the material, and it is the relative permittivity of any material at zero frequency. The electrostatic properties, for instance capacitance charge, screening and energy storage capability can be characterized by the dielectric constant.

Real and imaginary part of dielectric constant with frequency for different strain values are given in Fig. 4a and b. For $\mathrm{ZnO}$, the dielectric constant is varied by applying strain from $-5 \%$ to $5 \%$ as given in Fig. 3a. Dielectric constant is gradually increased under applied uniaxial strain. Step decrease of $\varepsilon_{1}(\omega)$ around $1 \mathrm{eV}$ is obtained for zero strain $\mathrm{ZnO}$, which goes toward zero, then winds up negative and in last achieves a minimum as appeared in Fig. 4a. For uniaxial strain $(-5 \%)$ system as well as $(5 \%)$ system almost steady steep decrease in $\varepsilon_{1}(\omega)$ is observed.

Dielectric loss $\varepsilon_{2}(\omega)$ of the $\mathrm{ZnO}$ and $\mathrm{ZnO}$ under uniaxial strain are introduced in Fig. 4b. As indicated by the electric change hypothesis of dielectric reaction, the low-energy edge of $\varepsilon_{2}(\omega)$ is concerned with the selection rules of electric transition in optical one-photon process. Electron-photon coupling can prompt transition between occupied and unoccupied states and so beginning stage of the native optical response ought to be larger than that band gap. Dielectric function $\varepsilon_{2}(\omega)$ is plotted for energy range of $1-5 \mathrm{eV}$. Steep increase in $\varepsilon_{2}(\omega)$ and peaks at little bit different values of energies are obtained for $\mathrm{ZnO}$ and uniaxial strained $\mathrm{ZnO}$ films. 


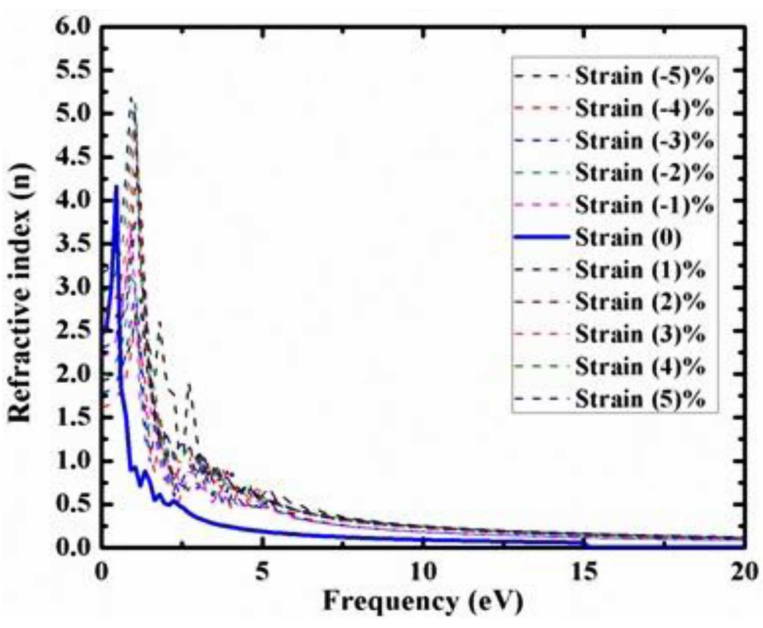

Fig. 5. Variations of refractive index $n$ with frequency energy under different uniaxial strain ( $-5 \%$ to $5 \%$ ).

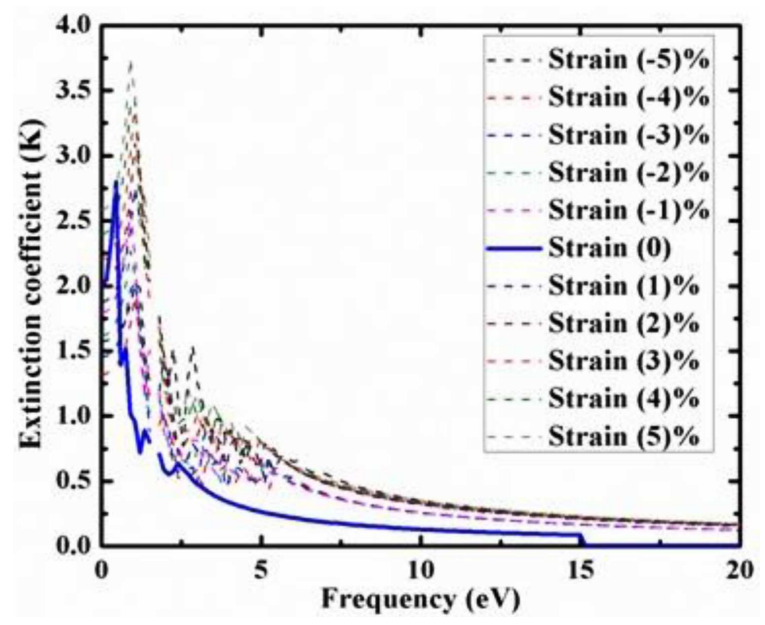

Fig. 6. Variations of extinction coefficient $k$ with frequency energy under different unaxial strain $(-5 \%$ to $5 \%)$.

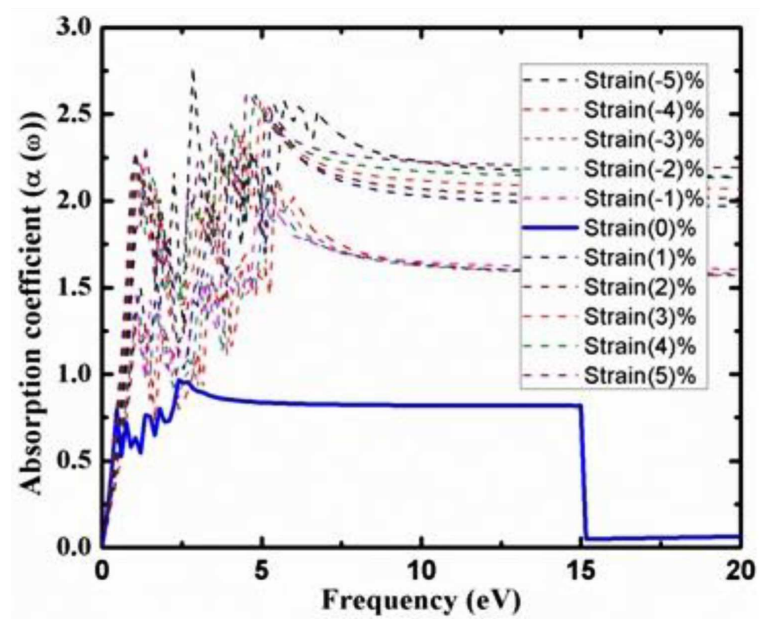

Fig. 7. Variations of absorption coefficient $\alpha(\omega)$ with frequency energy under different strain ( $-5 \%$ to $5 \%)$.

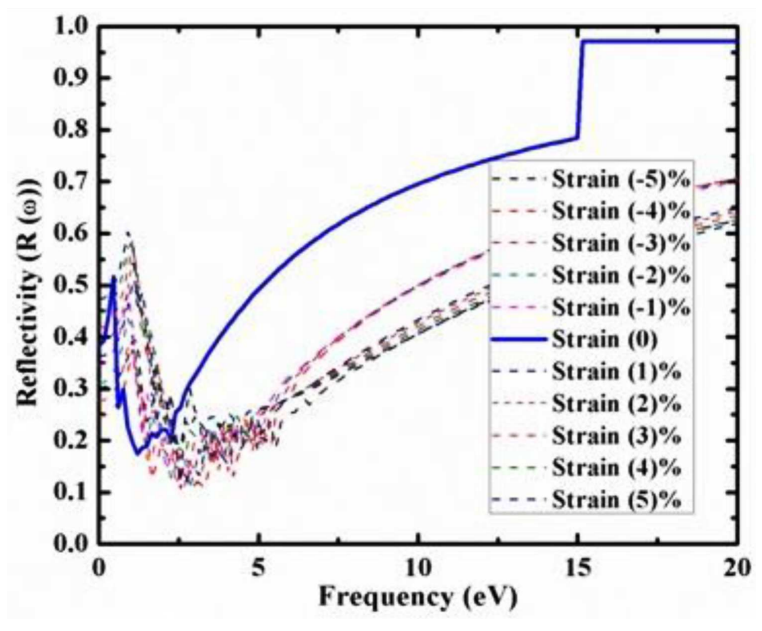

Fig. 8. Variations of reflectivity $R(\omega)$ with frequency energy under different strain ( $-5 \%$ to $5 \%)$.

Figures 5 and 6 represent variation of refractive index and extinction coefficient with frequency under different uniaxial strain of $\mathrm{ZnO}$. Refractive index is 2.5 for energy equal to zero. In case of $\mathrm{ZnO}$, the refractive index values are observed between 1.5 to 2.5 and are almost constant for strain from $-5 \%$ to $5 \%$. Wide of range refractive index has great importance. $\mathrm{ZnO}$ has various applications in optical devices, ophthalmic devices, nanodevices, semiconductors, light weight and polymeric materials which are used in solar cells and in antireflective coatings. The maximal estimation of the extinction coefficient $k$ is situated at 2.8 corresponding to the zero at frequency energy. The structure of the imaginary part of the dielectric function matches to the structure of the extinction coefficient, and increase in the slight uniaxial strain $(-5 \%$ to $5 \%$ ) raises extinction coefficient corresponding to the frequency energy.

The calculated spectra of the absorption coefficient $\alpha(\omega)$, reflectivity coefficient $R(\omega)$, are shown in Figs. 7 and 8, respectively. The absorption coefficients of $\mathrm{ZnO}$ under uniaxial strain condition are plotted in Fig. 7. Absorption coefficient of unstrained $\mathrm{ZnO}$ is varied from 0.5 to 1 whereas this value is varied from 0.5 to 2.7 under uniaxial strain ( $-5 \%$ to $5 \%$ ). Therefore, absorption coefficient is increased under uniaxial strain.

The reflectivity coefficient of $\mathrm{ZnO}$ and $\mathrm{ZnO}$ under uniaxial strain is shown in Fig. 8. It has a value of 0.4 for the zero frequency limit as mentioned in Fig. 8. The reflectivity coefficient increases with increasing frequency energy to reach a maximum value of about 0.8 for frequency energy of about $15 \mathrm{eV}$.

\section{Conclusion}

This work reported DFT studies for the band structures, band gap and optical properties of $\mathrm{ZnO}$ used by optimization of the geometry and calculation using firstprinciples calculations. In addition to this the band gap 
of $\mathrm{ZnO}$ was tuned continuously under uniaxial strain $(-5 \%$ to $+5 \%$ interval $)$. By using the tight binding approximation, we have calculated the electronic dispersions of these systems. Dielectric constant, refractive index, absorption coefficient were evaluated using Green's functions. Lattice constant $a$ and $c$ of $\mathrm{ZnO}$ were found to change linearly with uniaxial strain. The band gap was extended linearly with increment of uniaxial strain, and primarily contributed from strain in the $c$-axis direction. Dielectric loss decreased with the increasing uniaxial strain at low energy. For higher energy, imaginary part of dielectric function is nearly linear to the uniaxial strain and a blue shift occurred. Band gap of strained $\mathrm{ZnO}$ is larger than that of unstrained $\mathrm{ZnO}$, which provided a hypothetical base for the regulation of the band gap of $\mathrm{ZnO}$. It is strongly anisotropic, the band gap was continuously modified under the strain. The large and adjustable band gaps showed a great potential of applications in the future electronic devices. $\mathrm{ZnO}$ revealed semiconducting properties with a moderate band gap that can be modified by strain.

\section{References}

[1] Ü. Özgür, Y.I. Alivov, C. Liu, A. Teke, M.A. Reshchikov, S. Doğan, V. Avrutin, S.-J. Cho, and H. Morkoç, J. Appl. Phys. 98, 1 (2005).

[2] J. Wienke, B. van der Zanden, M. Tijssen, M. Zeman, Sol. Energy Mater. Sol. Cells 92, 884 (2008).

[3] Y.R. Ryu, J.A. Lubguban, T.S. Lee, H.W. White, T.S. Jeong, C.J. Youn, B.J. Kim, Appl. Phys. Lett. 90, 131115-3 (2007).

[4] S.J. Jiao, Z.Z. Zhang, Y.M. Lu, D.Z. Shen, B. Yao, J.Y. Zhang, B.H. Li, D.X. Zhao, X.W. Fan, Z.K. Tang, Appl. Phys. Lett. 88, 1 (2006).

[5] M. Murphy, S. Quinn, J. Young, P. Parkin, B. Taylor, Neurology 71, 1889 (2008).

[6] M.F. Alias, R.M. Aljarrah, H.K.H. Al-lamy, K.W. Adem, Int. J. Appl. Innovat. Eng. Manage. (IJAIEM) 2, 198 (2013).

[7] M. Goto, A. Kasahara, M. Tosa, T. Kimura, K. Yoshihara, Appl. Surf. Sci. 185, 172 (2002).

[8] M. Topsakal, S. Cahangirov, E. Bekaroglu, S. Ciraci, Phys. Rev. B 80, 235119 (2009).

[9] A.D. Torrez-Baptista, A. Fazzio, J.T. Arantes, Computat. Mater. Sci. 149, 91 (2018).

[10] R. Shao, K. Zheng, B. Wei, Y. Zhang, Y. Li, X. Han, Z. Zhang, J. Zou, Nanoscale 6, 4936 (2014).

[11] Y. Wang, W. Tang, J. Zhu, J. Liu, Computat. Mater. Sci. 99, 145 (2015).

[12] X.C. Liu, Y.J. Ji, J.Q. Zhao, L.Q. Liu, Z.P. Sun, H.L. Dong, Acta Phys. Sin. 59, 4925 (2010).

[13] Y.F. Li, B. Yao, Y.M. Lu, Z.P. Wei, Y.Q. Gai, C.J. Zheng, B.H. Li, Z.P. Wei, D.Z. Shen, X.W. Fan, L. Xiao, S.C. Xu, Y. Liu, Appl. Phys. Lett. 91, 232115-3 (2007).
[14] L. Zhang, G.F. Ji, F. Zhao, Z.Z. Gong, Chin. Phys. B 20, 047102-7 (2011).

[15] C. Adenis, V. Langer, O. Lindqvist, Acta Crystallogr. C 45, 941 (1989).

[16] A. Bilic, J.R. Reimers, N.S. Hush, J. Chem. Phys. 122, 0947708-15 (2005).

[17] P.A. Dowben, Surf. Sci. Rep. 40, 151 (2000).

[18] L. Hedin, Phys. Rev. 139, A796 (1965).

[19] J.P. Perdew, in: Electronic Structure of Solids '91, Eds. P. Ziesche, H. Eschrig, Academic, Berlin 1991, p. 11.

[20] M.K. Yaakob, M.F.M. Taib, M.S.M. Deni, A. Chandra, L. Lu, M.Z.A. Yahya, Ceram. Int. 39, S283 (2013).

[21] S.L. Dudarev, G.A. Botton, S.Y. Savrasov, C.J. Humphreys, A.P. Sutton, Phys. Rev. B 57, 1505 (1998).

[22] A.D. Becke, J. Chem. Phys. 98, 1372 (1993).

[23] G. Kotliar, D. Vollhardt, Phys. Today 57, 53 (2004).

[24] V.I. Anisimovy, F. Aryasetiawanz, A.I. Lichtenstein, J. Phys. Condens. Matter 9, 767 (1997).

[25] M.K. Yaakob, N.H. Hussin, M.F.M. Taib, T.I.T. Kudin, O.H. Hassan, A.M.M. Ali, M.Z.A. Yahya, Integrat. Ferroelectr. 155, 15 (2014).

[26] R. Chowdhurya, P. Rees, S. Adhikari, F. Scarpa, S.P. Wilks, Physica B 405, 1980 (2010).

[27] B. Hammer, L. Hansen, J. Nørskov, Phys. Rev. B 59, 7413 (1999).

[28] X.H. Peng, A. Alizadeh, S.K. Kumar, S.K. Nayak, Int. J. Appl. Mech. 01, 483 (2009).

[29] J.P. Perdew, M. Levy, Phys. Rev. Lett. 51, 1884 (1983).

[30] M.D. Segall, P.J.D. Lindan, M.J. Probert, C.J. Pickard, P.J. Hasnip, S.J. Clark, M.C. Payne, J. Phys. Condens. Matter 14, 2717 (2002).

[31] R. Ghosh, D. Basak, S. Fujihara, J. Appl. Phys. 96, 2689 (2004).

[32] Y. Ping, P. Li, Z. Li-Qiang, W. Xiao-Liang, W. Huan, S. Xi-Fu, X. Fang-Wei, Chin. Phys. B 21, 016803-5 (2012).

[33] Y.F. Li, B. Yao, Y.M. Lu, C.X. Cong, Z.Z. Zhang, Y.Q. Gai, C.J. Zheng, B.H. Li, Z.P. Wei, D.Z. Shen, X.W. Fan, L. Xiao, S.C. Xu, Y. Liu, Appl. Phys. Lett. 91, 1 (2007).

[34] C.G. van de Walle, R.M. Martin, Phys. Rev. Lett. 62, 2028 (1989).

[35] B. Adolph, J. Furthmüller, F. Bechstedt, Phys. Rev. B 63, 125108-8 (2001).

[36] G. Kresse, D. Joubert, Phys. Rev. B 59, 1758 (1999).

[37] F. Tran, P. Blaha, Phys. Rev. Lett. 102, 226401 (2009). 